\title{
The Mobil Type Weather Information Detects Device
}

\author{
Fa-Ta Tsai ${ }^{1{ }^{* *}, \text { Wan-Yi Lu }}{ }^{2}$, Rwei-Ching Chang ${ }^{3}$, Ching-Kong Chao ${ }^{1}$ \\ ${ }^{1}$ Department of Mechanical Engineering, National Taiwan University of Science and Technology, Taiwan \\ ${ }^{2}$ Graduate Institute of Applied Science and Technology, National Taiwan University of Science and Technology, Taiwan \\ ${ }^{3}$ Department of Mechanical and Computer Aided Engineering, St John's University, Taiwan
}

Copyright $\bigcirc 2015$ by authors, all rights reserved. Authors agree that this article remains permanently open access under the terms of the Creative Commons Attribution License 4.0 International License

\begin{abstract}
In the case, the system proposed a simple solar powered weather information gathering module, which can be mounted on mobile vehicles, using green energy, Global Positioning System (GPS) and information and communications technology (ICT). When parking in the outdoor, the system operate and the sensor switch too. All the measurement data, not only can provide the real-time local temperature and humidity information to the stranger, who will go to here, because it will detect than the automatic observation station more accurate and more instant. According to the GPS data, it will be uploaded to public homepage, or the cloud database computing parameters, all as an effort to make contribution in the search of new energy resources. Overall the weather information gathering mode uses Android App software, integrated with the image capture function of smart phone and GPS function. Through this system the spindle in the "real-time video transmission system" and "Journey Log"combine to provide more information to integrally analyze and instantly display the weather information of various regions.
\end{abstract}

Keywords Information and Communications Technology(ICT), Global Positioning System(GPS), Cloud Database

\section{Introduction}

Due to the overuse of fossil energy, the global greenhouse effect becomes more serious, and abnormal weathers are more common. The public has realized that earth resources have been exploited, the natural environment should be treated well during production and manufacturing. Under this common understanding, the enterprises are developing and designing new products with environmentally friendly material and green energy. Energy saving and carbon reduction, using green energy (solar power generation, wind electric power generation, temperature difference power generation) have been the present trend. Due to the overuse of fossil energy, the global greenhouse effect becomes more serious, and abnormal weathers are more common. The public has realized that earth resources have been exploited, the natural environment should be treated well during production and manufacturing. Under this common understanding, the enterprises are developing and designing new products with environmentally friendly material and green energy. Energy saving and carbon reduction, using green energy (solar power generation, wind electric power generation, temperature difference power generation) have been the present trend.

The rapid development of Internet and communication technology contributes to successive emergence of products of ICT [1-5]. The most representative device integrating information and communication technology is the smart phone. It contributes to the mass production and popularization of smart phones and tablets, brining great changes to daily life. It is now prevalent to have a smart phone, allowing convenient access to the information via the Internet. It was unimaginable just two decades ago, until the ICT was developed.

The smart phone becomes more powerful, as it is equipped with high-definition display screen, Multitouch, high resolution camera, GPS (Global Positioning System), 3G wireless communication, WiFi (Wireless Fidelity), Bluetooth wireless communication and programmer control and editing development functions. The App software becomes increasingly diversified. The information is easily accessible via the Internet. This era is also called the era of information explosion. The development of ICT has brought great changes to our life.

\section{Composition and Mode of Operation of the System}

\subsection{Solar Powered Weather Information Gathering Module System Architecture}

The solar powered weather information gathering module proposed in this study is embedded with precision temperature sensor, humidity sensor and Bluetooth 
information transmission. It can be equipped with weather information gathering components as required, such as UV intensity sensor, air quality sensor, air pressure sensor and so on. The module is powered by lithium battery and solar cell. It can be connected to external mobile power supply or to charge mobile vehicle. The photo of the module is shown in Figure 1.

The overall conceptual framework of this design is shown in Figure 2. The solar powered weather information gathering module is mounted on the mobile vehicle. The solar panel of green energy supplies the module and mobile vehicle with auxiliary charging power. The module uses the mobile vehicle's battery power at night. The module detects weather information for the use by smart phones, and then integrates and transmits the information data to the cloud database, so as to provide real-time weather information for other information users.

The smart phone is installed with exclusive App software in the procedure. The meteorological data of air temperature and humidity collected by the solar powered weather information gathering module are transmitted by the Bluetooth transmitter inside the module to the smart phone instantly. The schematic diagram is shown in Figure 3.

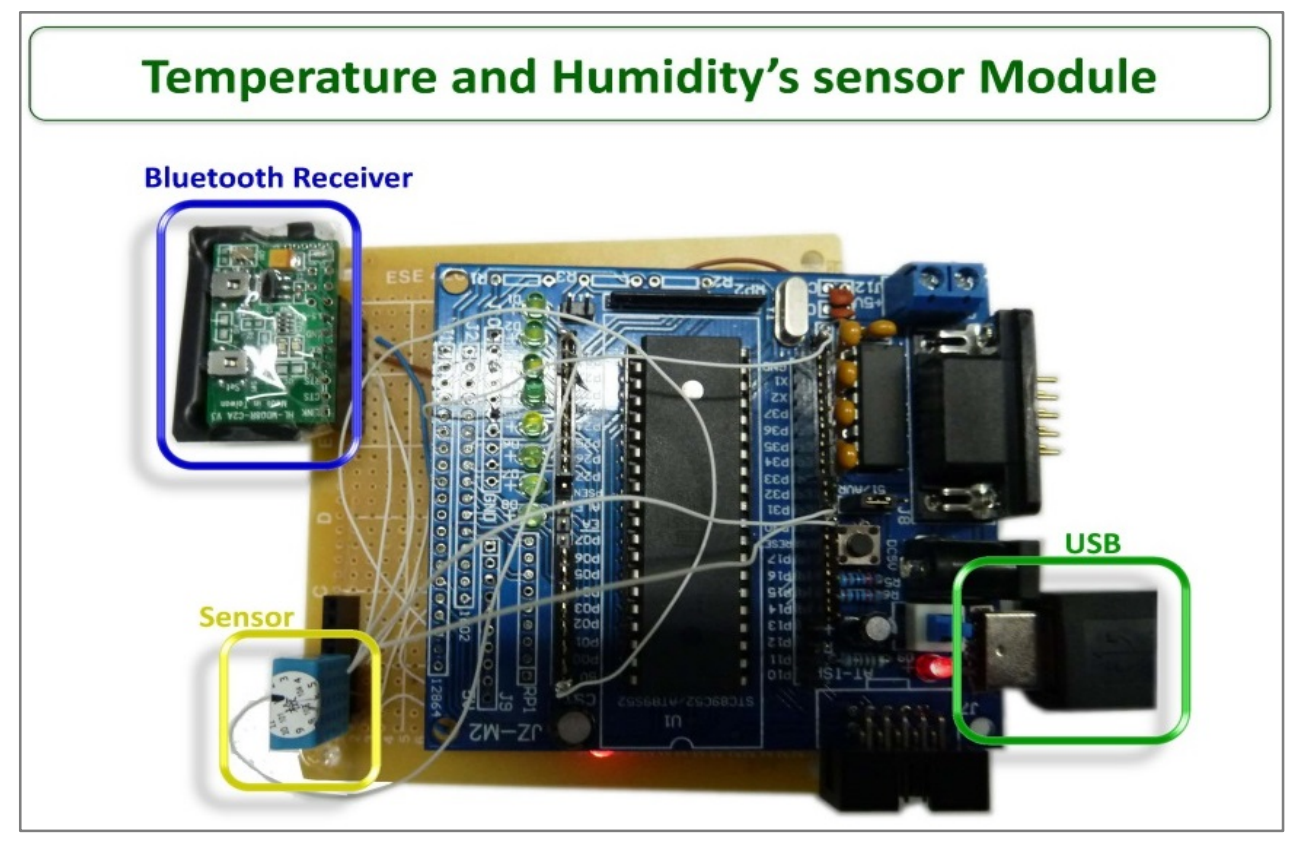

Figure 1. Solar powered weather information gathering module

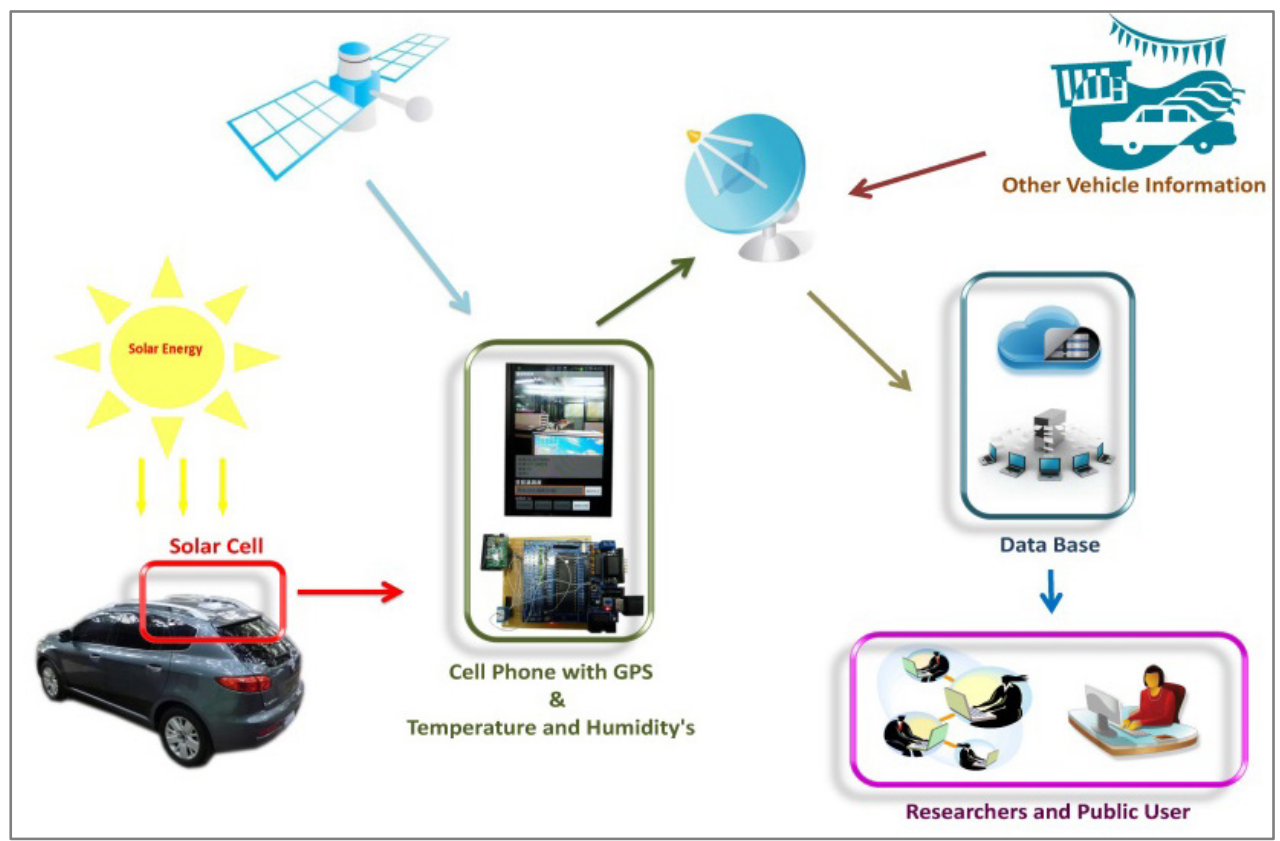

Figure 2. Overall conceptual framework of design 


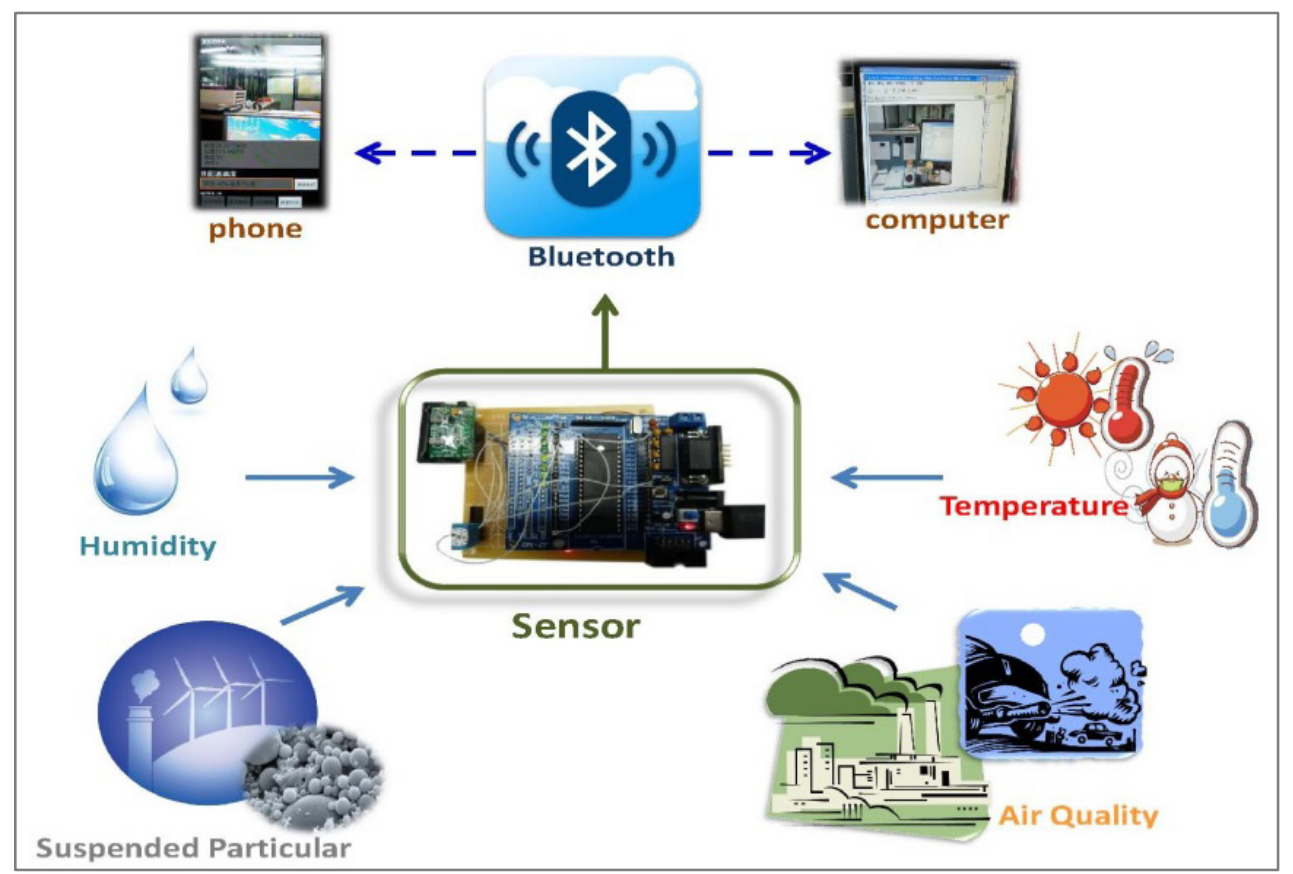

Figure 3. Schematic diagram of module and mobile phone data transmission

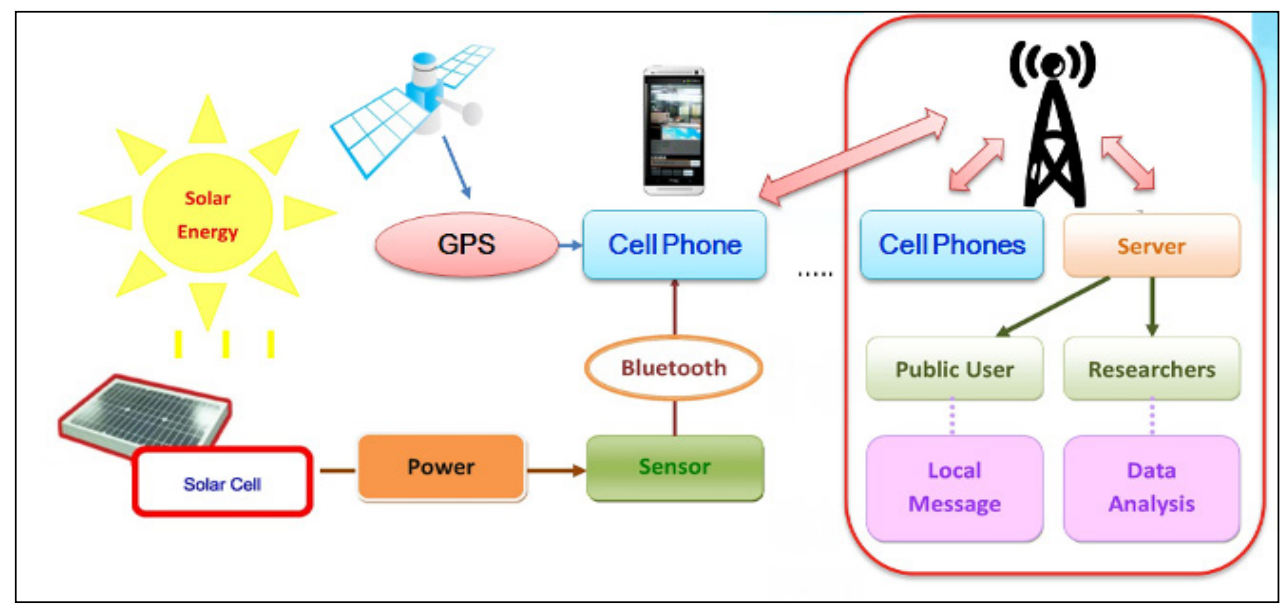

Figure 4. Flow chart of gathering and display of weather information of various regions

Afterwards, the mobile phone integrates the received real-time weather information, GPS positioning information and image capture function inside the mobile phone, the GPS coordinates, and the existing photo information. It then uploads the information to the cloud database by using $3 \mathrm{G}$ mobile communication. When numerous users use this weather detection module, the database will have numerous real-time data. After analysis, these data are displayed to other users who need the information instantly. The flow chart of global concept is shown in Figure 4.

Another function is the track positioning of mobile vehicle. The App software captures the GPS coordinates, the locating point is combined with Google Map to display the movement track and time of vehicle. It guards against theft, further protecting the vehicle.

\subsection{Actual Test for Solar Powered Weather Information Gathering Module}

The smart phone opens the weather information gathering application program, then the mobile phone opens the Bluetooth transmission protocol. The weather information returned from the solar powered weather information gathering module is received via Bluetooth-based two-way transmission, and displayed on the screen of mobile phone. The App software integrates the GPS latitude and longitude coordinates signals with image capture function of mobile phone, to upload the field image, GPS coordinates and weather information via $3 \mathrm{G}$ mobile network to the cloud database system. This App program can be executed in background, timing data access and transmission. The image capture function can be set and cancelled. The mobile phone-side APP software function menu is shown in Figure 5.

The mobile vehicle was driven from St. John's University towards Fu-Guei-Jiao Lighthouse. After testing verification, the cloud database integrates various data uploaded from 
smart phone client-sides in different places with Google Map tracks according to time for drawing, and analyzes and displays the collected data on the browser. The users can search for the weather information (e.g. temperature, humidity, air pressure, etc.) of various places on the Internet. The cloud database can analyze the running track, current and the last location of a user individually to protect the owner against theft.

Figure 6 shows the first movement track point of vehicle at St. John's University and meteorological data recorded in the cloud database. Figure 7 shows the last movement track point at Fu-Guei-Jiao Lighthouse and meteorological data.

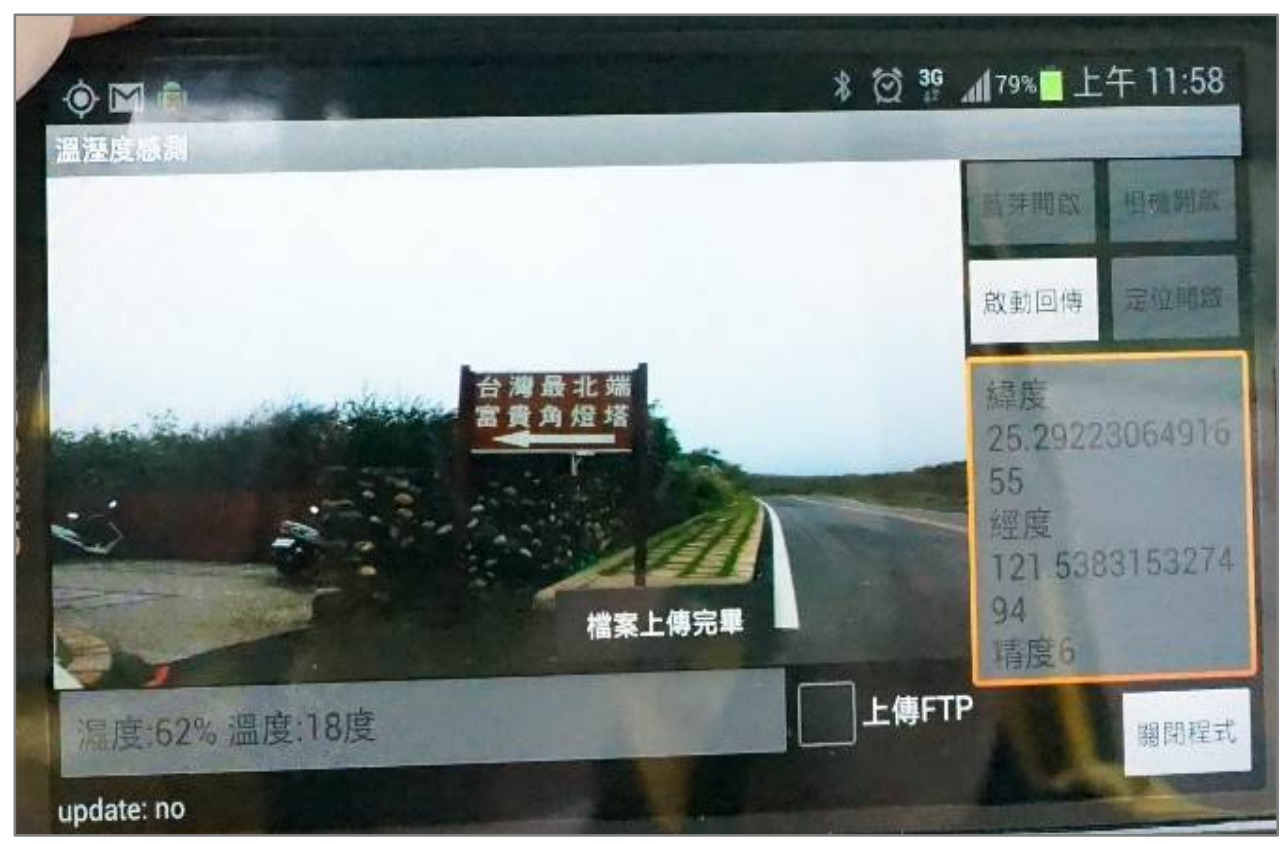

Figure 5. Smart phone-side App weather information gathering software menu

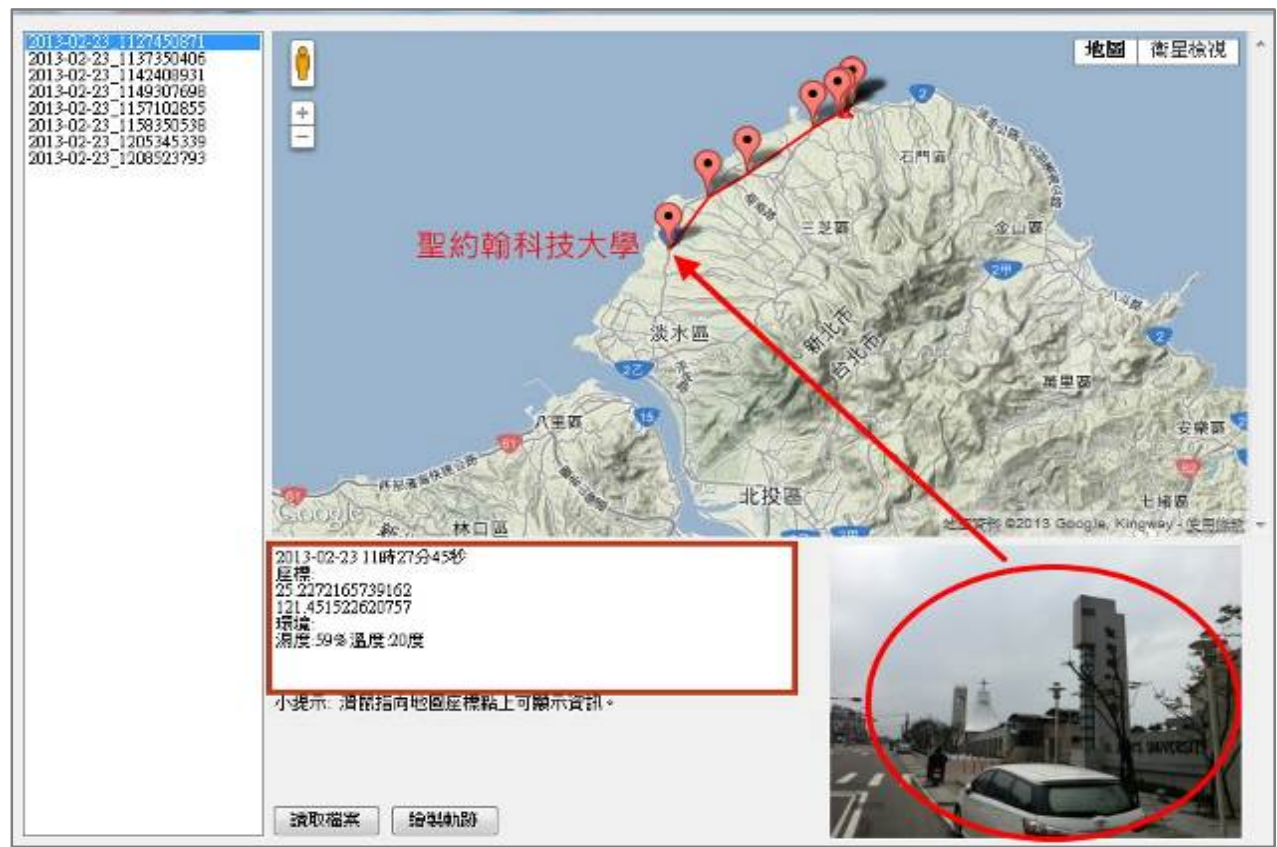

Figure 6. The first movement track point of vehicle and meteorological data recorded in cloud database 


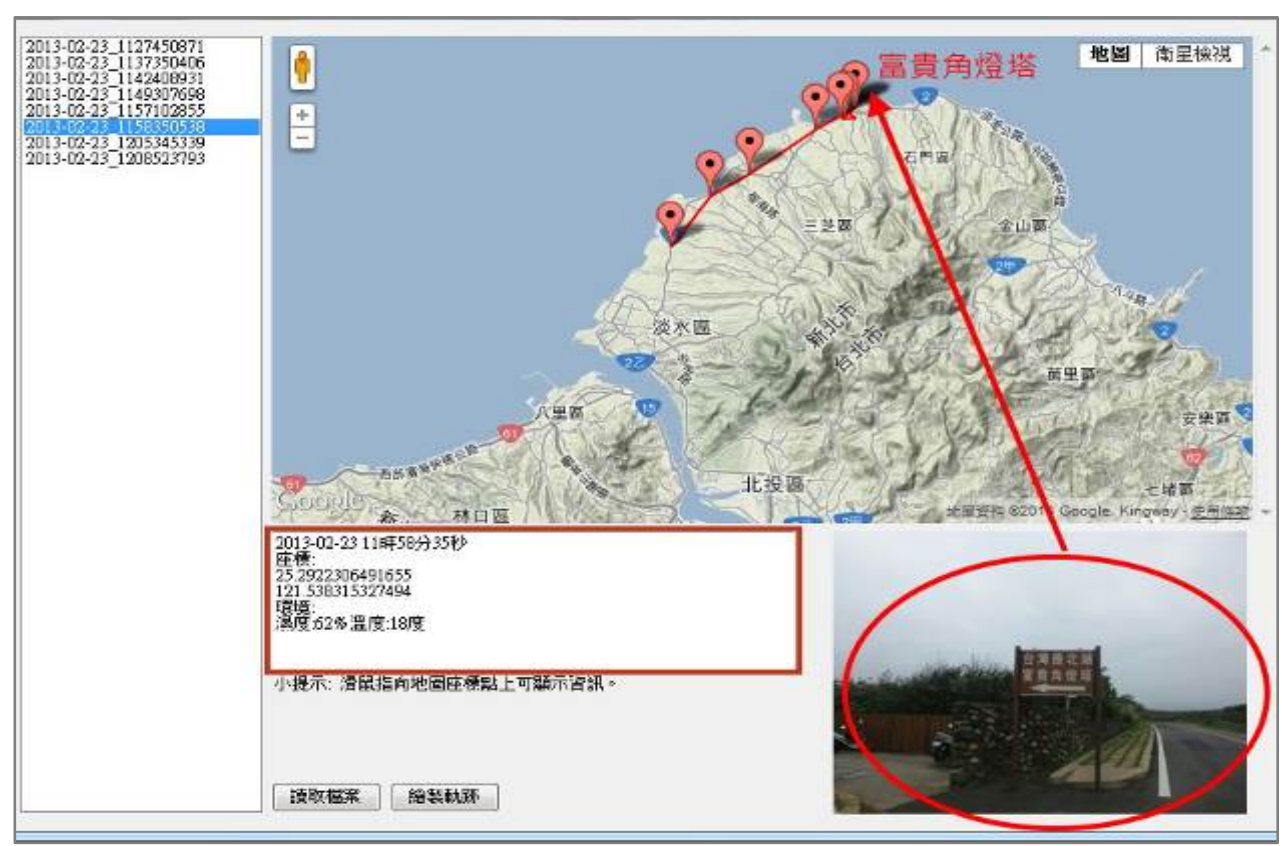

Figure 7. The last movement track point of vehicle and meteorological data recorded in cloud database

\subsection{Characteristics of Solar Powered Weather Information Gathering Module}

Characteristics of solar powered weather information gathering module are explained as follow.

(1) The solar powered weather information gathering module can collect air temperature and humidity. It can be equipped with other meteorological sensors, such as UV intensity, air pressure, and wind strength, to provide diversified weather information.

(2) The solar powered weather information gathering module can be connected to smart phone. The weather information is transmitted via Bluetooth to the mobile phone instantly, and displayed.

(3) The solar powered weather information gathering module uses solar panel of green energy. It is charged automatically during the day, and it assists the mobile vehicle charging.

(4) The weather information gathering APP software integrates the weather information with GPS coordinates and actual photo of the existing circumstances, and uploads the data to the cloud database instantly to reflect the existing circumstances.

(5) The weather information gathering APP software can be executed automatically in the background, transmitting weather information to the cloud database automatically.

(6) The cloud database can integrate the existing weather information instantly for the users to inquire about the weather information.

(7) The cloud database can integrate the historical movement tracks of mobile vehicles, so as to obtain the direction of mobile vehicles, and to further analyze the meteorological data of various places at different time points.

(8) The cloud database can integrate historical tracks with Google Map to protect the owner against theft.

\section{Conclusions and Future Work}

The solar powered weather information gathering module proposed in this study is combined with the functions and ideas of green energy and ICT. It has very high practicability and development potential in the future. Based on the integration of smart phone, App software, mobile communication and cloud database, the weather information of various regions is collected instantly. Related data can be provided for researchers to monitor weather environment, and the real-time weather information can be provided for common people as reference frame of daily life. The mobile vehicles can be tracked and located. Based on the GPS coordinates captured by the App software, the locating point is combined with Google Map to display the movement track and time of vehicles to protect the owners against theft, further protecting the vehicles.

In the future, this type of module can be provided with other functions. The new ICT can be integrated into vehicles through direct cooperation with automobile factories, such as $4 \mathrm{G}$ communication and real-time vehicle's condition analysis, so as to provide more perfect information gathering and analysis and convenient mobile communication. Further research and development will be carried out in the future.

\section{REFERENCES}

[1] Q. X. Meng and M. Z. Li, New Economy and ICT 
development in China, Information Economics and Policy, 14, pp. 275-295, 2002.

[2] R. Roman and R. D. Colle, Content creation for ICT development projects: Integrating normative approaches and community demand, Information Technology for Development, 10, pp. 85-94, 2003.

[3] P. Constantinides and M. Barrett, Negotiating ICT development and use: The case of a telemedicine system in the healthcare region of Crete, Information and Organization, 16, pp. 27-55, 2006.

[4] S. Sassi and M. Goaied, Financial development, ICT diffusion and economic growth: Lessons from MENA region, Telecommunications Policy, 37, pp. 252-261, 2013.

[5] S. Miyazaki, H. Idota and H. Miyoshi, Corporate productivity and the stages of ICT development, Information Technology and Management, 13, pp. 17-26, 2012.

[6] T. Ojanpera and R. Prasad, An Overview of Air Interface Multiple Access for IMT2000/UMTS, IEEE Communications Magazine, 36, pp. 82-95, 1998.

[7] D. Kaczman, M. Shah, M. Alam, M. Rachedine, D. Cashen, L. Han and A. Raghavan, A Single-Chip 10-Band WCDMA/HSDPA 4-Band GSM/EDGE SAW-less CMOS Receiver With DigRF 3G Interface and $90 \mathrm{dBm}$ IIP2, IEEE JOURNAL OF SOLID-STATE CIRCUITS, 44, pp. 718-739, 2009.

[8] A. Ghosh, R. Ratasuk, B. Mondal, N. Mangalvedhe and T. Thomas, LTE-Advanced: Next-Generation Wireless Broadband Technology, IEEE Wireless Communications, 17, pp. 10-22, 2010. 